\title{
Increased C4 and decreased C3 levels are associated with a poor prognosis in patients with immunoglobulin $A$ nephropathy: a retrospective study
}

Min Pan ${ }^{1,2}$, Ji Zhang ${ }^{3}$, Zhanyuan Li², Lingwei $\mathrm{Jin}^{2}$, Yu Zheng², Zhihong Zhou², Su Zhen ${ }^{3}$ and Guoyuan Lu ${ }^{1 *}$

\begin{abstract}
Background: An association between serum complement levels and poor renal prognosis in patients with immunoglobulin A nephropathy (IgAN) remains controversial.

Methods: We conducted a retrospective study examining the relationship between serum complement levels and prognosis in patients with IgAN. Between 2009 and 2013, patients with biopsy-confirmed IgAN were identified from the Second Affiliated Hospital of Wenzhou Medical College, China, and various parameters were documented during follow-up until 2015. The definition of the primary endpoint was a decrease of estimated glomerular filtration rate (eGFR) more than $30 \%$ from their baseline levels.

Results: A total of 403 patients (55.3\% female, average 33.7 months of follow-up) were identified and enrolled, with the primary endpoint occurring in 39 (9.8\%) patients. Among the patients selected, 202 (50.1\%) received corticosteroid treatment alone or in combination with another immunosuppressant (GS group), while others did not receive immunosuppressive treatment (non-GS group). The incidence of the primary endpoint was slightly lower in the GS group compared to the non-GS group (7.0\% versus $12.6 \%, p=0.06)$. Multivariate Cox proportional-hazard regression analyses, adjusting for age, systolic and diastolic blood pressure, 24-h urine protein, and immunosuppressive therapy, showed that serum complement 4 (C4) levels (hazard ratio [HR] 2.4, 95\% confidence interval [Cl] 1.6-3.8, $p<0$. $001)$ and serum complement 3 (C3) levels (HR 0.6, 95\% Cl 0.2-0.6, $p<0.001$ ) were significantly associated with a poor prognosis among patients with IgAN.
\end{abstract}

Conclusions: We demonstrated that an increase in serum C4, as well as a decrease in C3, was an important outcome determinant for patients with IgAN. Testing serum C3 and C4 levels might assist in predicting renal outcomes among these patients.

Keywords: IgA nephropathy, Complement, Chronic kidney disease, Renal failure

\section{Background}

Immunoglobulin A nephropathy (IgAN) is the most common type of primary glomerulonephritis worldwide, and is characterized by the deposition of IgA in the glomerular mesangium [1,2]. The clinical course of IgAN is highly variable, ranging from asymptomatic disease with abnormal urinalysis findings, to rapidly progressive renal failure.

\footnotetext{
* Correspondence: lu_guoyuan@hotmail.com

'Department of Nephrology, The First Affiliated Hospital of Soochow

University, 88 Shizi St., Suzhou 215006, Jiangsu, People's Republic of China Full list of author information is available at the end of the article
}

Approximately one-third of patients with IgAN eventually develop end-stage renal disease (ESRD) within 20-30 years after their initial diagnosis [1].

Over the last two decades, risk factors for the progression of IgAN have been identified, including the presence of proteinuria, high blood pressure, and low estimated glomerular filtration rate (eGFR) at the time of renal biopsy. Findings from pathological examination are also found to be associated with renal outcomes; for example, a new grading system termed Oxford Classification of IgAN, as well as the MEST scoring system, 
attempt to score changes in different anatomical compartments including mesangium hypercellularity (M), endocapillary hypercellularity (E), segmental glomerulosclerosis (S), and tubular atrophy/interstitial fibrosis $(\mathrm{T})$. Results obtained using this classification system has been validated in a variety of studies for the prediction of renal outcomes $[3,4]$. However, the aforementioned parameters may fail to capture the degree of systemic inflammation, an important pathophysiologic contributor to IgAN.

IgAN is thought to arise due to autoimmunity, involving the aberrant activation of both the alternative and mannose-binding lectin (MBL) pathways. Complement activation at both glomerular and systemic levels plays a key role in the pathogenesis and the clinical presentations of IgAN [5-8]. Serum complement levels are surrogate markers of the degree of complement activation, and complement $3(\mathrm{C} 3)$ and complement $4(\mathrm{C} 4)$ are the most widely measured parameters during clinical practice. However, it remains unclear whether serum complement levels can predict outcomes in patients with IgAN. The main objective of this study was to investigate the relationship between serum C3 and C4 levels and the prognosis of patients with IgAN.

\section{Methods}

\section{Subjects}

Participants of this study were enrolled based on the following criteria: biopsy-proven primary IgAN diagnosed between 2009 and 2013; follow-up until 2015 in the Second Affiliated Hospital of Wenzhou Medical University, China; no history of corticosteroid or immunosuppressant treatment before renal biopsy. The exclusion criteria were as follows: patients demonstrating secondary causes of IgAN, such as systemic lupus erythematosus, Henoch-Schönlein purpura, hepatic disease, or ANCAassociated vasculitides. In addition, those with acute interstitial nephritis on renal biopsy, with severe comorbid diseases, including chronic infectious diseases, diabetic nephropathy, hypertensive nephropathy, or malignancy, were also excluded.

\section{Collection of clinical variables}

Demographic data (age and gender) and clinical features, including systolic and diastolic blood pressure (BP), body weight, body height, as well as serum creatinine, and 24-h urine protein levels were collected at the time of renal biopsy and during follow-up. Mean arterial pressure (MAP) was calculated as diastolic pressure plus one-third of the pulse pressure. Hypertension was defined according to a BP above 140/90 mmHg. Use of medications including immunosuppressant and antihypertensive medications (angiotensin-converting enzyme inhibitor [ACEI] and angiotensin receptor blocker [ARB]) were also recorded.
Results from renal biopsy results were graded according to the Oxford Classification of IgAN [9]. Serum creatinine was measured using an enzymatic assay (AU5831 Biochemistry Auto analyzer, Tokyo, Japan) at the time of biopsy, and eGFR was calculated using Chronic Kidney Disease Epidemiology Collaboration equation (CKD-EPI) [10]. Serum C3 (reference range $0.9-1.8 \mathrm{~g} / \mathrm{L}$ ) and C4 (reference range $0.1-0.4 \mathrm{~g} / \mathrm{L}$ ) levels were measured using a turbidimetric method by a commercial kit (Beckman Coulter IMMAGE, USA). 24-h proteinuria was quantified using a benzethonium chloride method by a biochemistry automatic analyzer (HITACHI 7180, Japan).

\section{Primary outcome}

Since the use of the eGFR doubling as the endpoint requires a longer follow-up period, Levey et al. proposed eGFR decline of $30 \%$ as an alternative surrogate endpoint in trails of CKD [11], The primary outcome of this study was defined as an eGFR decrease of more than $30 \%$ from the baseline level during follow-up.

\section{Statistical analysis}

Numerical variables were presented as mean (SD) or median with interquartile range (IQR). Variance homogeneity was tested by the Bartlett test. A Welch t-test was used for comparing homogeneous data, while a Wilcoxon rank test was used to compare heterogeneous data. A Kruskal-Wallis rank test was used for the comparison of multiple groups. Categorical variables were presented as percentages and compared by a $X^{2}$ test. Univariate and multivariate Cox proportional-hazards regression analysis was performed using the $c p h$ mathed of the rms package [12], and the default parameters of the program were used during the multivariate analysis. A nomogram was built for the prediction of three- and five-years survival rate. $P$-values were two-tailed and a value less than 0.05 were considered statistically significant. All analyses were performed using R-software (https://www.r-project.org) and graphics were drawn by ggplot2 [13].

\section{Results}

A total of 456 cases with primary IgAN were retrospectively identified, and 53 cases were excluded due to secondary $\operatorname{IgAN}(n=4)$, malignancy $(n=1)$, acute interstitial nephritis $(n=2)$, diabetic and hypertensive nephropathy $(n=5)$, infectious disease $(n=13)$, incomplete data $(n=6)$, and short follow-up time ( $<6$ months) $(n=22)$. Finally, 403 patients were included in this study.

\section{Baseline clinical, histopathological features, and follow-up results}

Patients' clinical and histopathological features at the time of renal biopsy are shown in Table 1 . The mean age 
Table 1 Clinical characteristics and the Oxford IgAN classification at the time of renal biopsy in 403 patients with IgAN

\begin{tabular}{|c|c|c|c|}
\hline \multicolumn{2}{|l|}{ Clinical characteristics } & \multicolumn{2}{|c|}{ Oxford classification (n [\%]) } \\
\hline Female (n [\%]) & $223(55.3)$ & \multicolumn{2}{|c|}{ Mesangial hypercellularity } \\
\hline Age (years, mean [SD]) & $37.1(11.8)$ & Mo & $280(69.4)$ \\
\hline Systolic blood pressure (mmHg, mean [SD]) & $129.7(18.1)$ & M1 & $121(30.0)$ \\
\hline Diastolic blood pressure (mmHg, mean [SD]) & $81.4(12.1)$ & \multicolumn{2}{|c|}{ Endocapillary hypercellularity } \\
\hline MAP (mmHg, mean [SD]) & $97.6(13.4)$ & E0 & $274(68)$ \\
\hline Hemoglobin (g/L, mean [SD]) & 125.9 (1 9.0) & E1 & $127(31.5)$ \\
\hline Serum creatinine $(\mu \mathrm{mol} / \mathrm{L}$, mean $[\mathrm{SD}])$ & $87.7(46.3)$ & \multicolumn{2}{|c|}{ Segmental glomerulosclerosis } \\
\hline eGFR (ml/min/1.73 m2, mean [SD]) & $94.5(30.9)$ & so & $101(25.1)$ \\
\hline $24 \mathrm{~h}$ urinary protein ( $\mathrm{g} / \mathrm{L}$, median $[\mathrm{IQR}])$ & $1.2(1.5)$ & S1 & $300(74.4)$ \\
\hline Serum C3 (g/L, mean [SD]) & $0.95(0.19)$ & \multicolumn{2}{|c|}{ Tubular atrophy/interstitial fibrosis } \\
\hline \multirow[t]{3}{*}{ Serum C4 (g/L, mean [SD]) } & $0.22(0.07)$ & T0 & $294(73.0)$ \\
\hline & & $\mathrm{T} 1$ & $84(20.8)$ \\
\hline & & $\mathrm{T} 2$ & $23(5.7)$ \\
\hline
\end{tabular}

$S D$ standard deviation, $I Q R$ interquartile range

was 37.1 (11.8) years; however, there was a small peak at the age of 58 . Approximately $32.6 \%$ of patients had hypertension at the time of renal biopsy, and 58.3, 26.1, 13.6, 1.2 and $0.7 \%$ patients had stage $1,2,3,4$, and 5 chronic kidney diseases (CKD), respectively. The followup characteristics are shown in Table 2. The mean duration of follow-up was 33.7 (16.6) months after renal biopsy, and $9.8 \%(n=39)$ developed the primary outcome, in which 16 patients developed end stage renal disease. $202(50.1 \%)$ received corticosteroid treatment alone or in combination with other immunosuppressant (GS group), while others received no immunosuppressant treatment (non-GS group). Among this cohort, proteinuria and serum creatinine levels were significant higher in the GS group than those in the non-GS group (for proteinuria, $1.56 \mathrm{~g} /$ day [0.94-3.03] versus $0.9 \mathrm{~g} /$ day [0.51.2 ], $p<0.001$; for serum creatinine, $83 \mu \mathrm{mol} / \mathrm{L}$ [59.5107.2] versus $75 \mu \mathrm{mol} / \mathrm{L}$ [57-95], $p=0.02)$. Most patients received ACEIs or ARBs for blood pressure control and for the reduction of proteinuria.

Table 2 Clinical characteristics of participants at the end of follow-up

\begin{tabular}{|c|c|}
\hline Clinical characteristics & \\
\hline Duration of follow-up (months, median [IQR]) & $35(11)$ \\
\hline Serum creatinine $(\mu \mathrm{mol} / \mathrm{L}$, median $[\mathrm{IQR}])$ & $78(41)$ \\
\hline UPCR (g/g, median [IQR]) & $0.5(1.0)$ \\
\hline eGFR $\left(\mathrm{ml} / \mathrm{min} / 1.73 \mathrm{~m}^{2}\right.$, mean $\left.[\mathrm{SD}]\right)$ & $91.1(34.7)$ \\
\hline $\operatorname{ESRD}(n[\%])$ & $16(4.0)$ \\
\hline Endpoint event (n [\%]) & $39(9.8)$ \\
\hline Immunosuppressive therapy (n [\%]) & $202(50.1)$ \\
\hline ACEI/ARB (n [\%]) & $255(87.3)$ \\
\hline
\end{tabular}

$S D$ standard deviation, IQR interquartile range
The association between serum complement levels and eGFR during follow-up

We next performed linear regression to investigate whether serum complement levels at the time of renal biopsy were associated with renal function at baseline or at the end of follow-up. There was no significant association between serum C3 levels and eGFR values at renal biopsy (regression coefficient, $-4.95, p=0.57$ ) or between serum C3 levels and eGFR at the end of follow-up (regression coefficient, $-1.36, p=0.89$ ). However, we observed a significant association between serum C4 levels and eGFR values at renal biopsy (regression coefficient, -171.5, $p<0.001$ ) and between serum C4 levels and eGFR values at the end of follow-up (regression coefficient, -163.2, $p<0.001$ ) (Fig. 1).

\section{The association between serum complement levels and histopathological features}

We used the Oxford Classification to categorize the pathological findings from all participants, and compared serum complement levels between different groups to evaluate the relationship between complement levels and histopathological features (Fig. 2). We found no association between serum C3 levels and scores using the Oxford classification, however serum C4 levels were significantly associated with scoring of tubular lesions (coefficient $0.27, p<0.001$ ) and segmental glomerulosclerosis (coefficient $0.12, p=0.02$ ).

\section{Risk factors for the prediction of poor renal prognosis} We performed univariate and multivariate Cox proportional-hazards regression analyses (Table 3). In the univariate analysis, serum C3 (hazard ratio 0.6, 95\% confidence interval $0.4-0.9, p=0.03$ ), $\mathrm{C} 4$ (hazard ratio $1.5,95 \%$ confidence interval $1-2.2, p=0.03$ ), as well as 


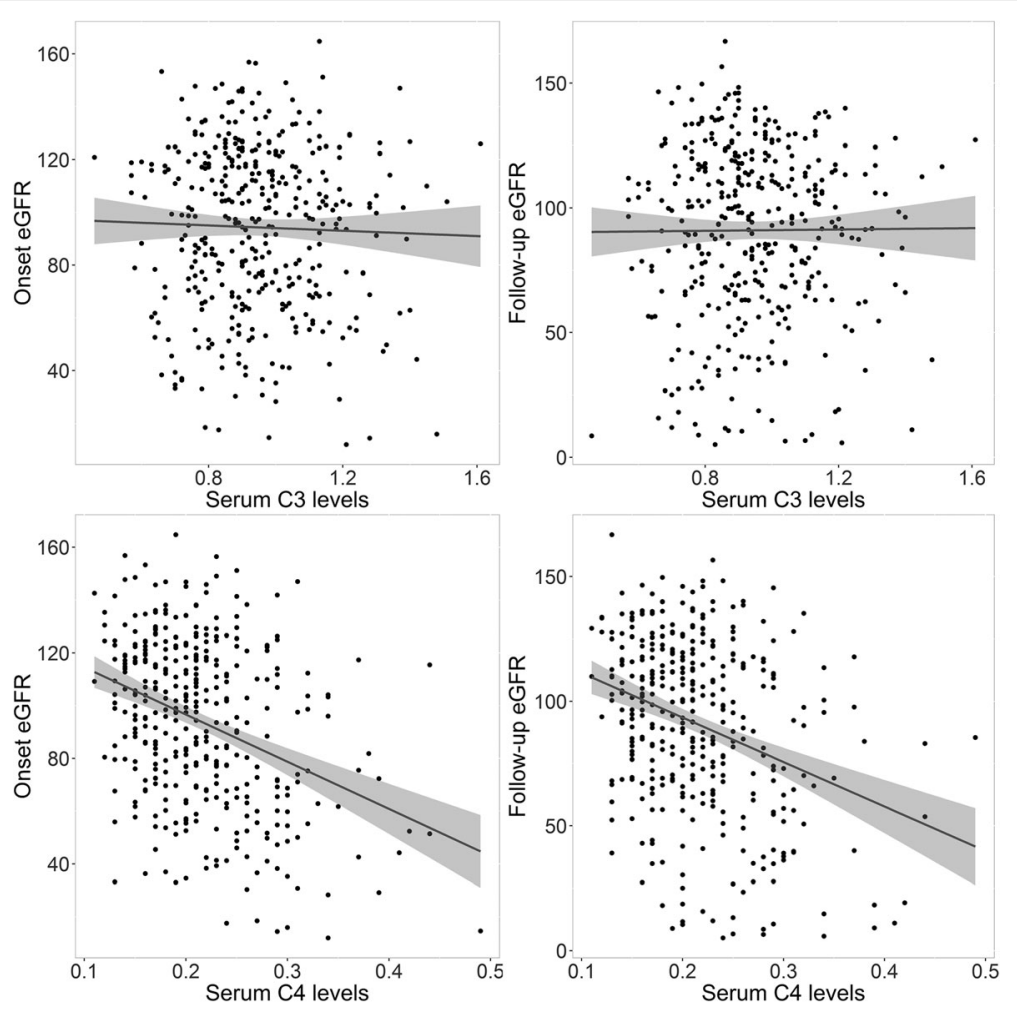

Fig. 1 Result from the linear regression analysis of the relationship between serum complement levels and eGFR values. Linear fitting showed that there was no significant correlation between serum C3 levels and eGFR values. Analyses also demonstrated that higher serum C4 levels were associated lower values of eGFR. Grey area outside the fitted line was the confidence interval of the fitted value

age, systolic and diastolic blood pressure, 24-h urine protein, and immunosuppressive therapy were associated with a worse renal prognosis among patients with IgAN. Including those variables with $P$ values less than 0.05 in the univariate analysis, multivariate analyses demonstrated that serum C3, C4, as well as age, 24-h urine protein, and immunosuppressive therapy were independent risk factors for renal outcome. For visualization of the role of these risk factors in predicting poor renal outcomes, all variables in the multivariate models were used to construct a nomogram for estimating three- and five- year survival (Fig. 3).

\section{Discussion}

Although risk factors for poor prognosis in patients with IgAN have been reported previously $[1,2]$, it is still difficult to accurately estimate renal prognosis in these patients. Although serum levels of $\mathrm{C} 3$ and $\mathrm{C} 4$ frequently fluctuate within the normal range in IgAN patients, studies have increasingly focused on the role of complement in the pathogenesis of IgAN [14-17]. However, the relationship between serum complement levels and the renal prognosis of patients with IgAN remains controversial.
There were studies discussing the relationship between serum C3 levels and the prognosis of IgAN. Kim et al. reported that low serum $\mathrm{C} 3$ at the time of renal biopsy was an independent risk factor for poor prognosis [18]. Lbels et al. also found that higher serum $\mathrm{C} 3$ at the end of follow up were associated with adverse outcomes, but lower baseline serum C3 levels were unrelated to prognosis [19]. Conversely, Komatsu et al. did not identify any difference in serum C3 levels between patients with severe and mild histopathological lesions, and further suggested that the serum IgA to C3 ratio, rather than C3 levels alone, might be a more appropriate marker for renal outcomes in patients with IgAN [20]. Similarly, another study comparing complement levels between IgAN patients and healthy volunteers did not reveal differences in serum C3 levels [21]. Few have specifically focused on the relationship between $\mathrm{C} 4$ and the prognosis of patients with IgAN. Lbels et al. found an independent association between serum $\mathrm{C} 4$ levels and patient outcomes, suggesting that these parameters reflect the severity of chronic inflammation [19]. Furthermore, Zhu et al. found that higher $\mathrm{C} 4$ levels at the time of renal biopsy were associated with a greater degree of renal injury, including lower eGFR, higher MAP and 24-h urinary protein, and more severe pathological features, 


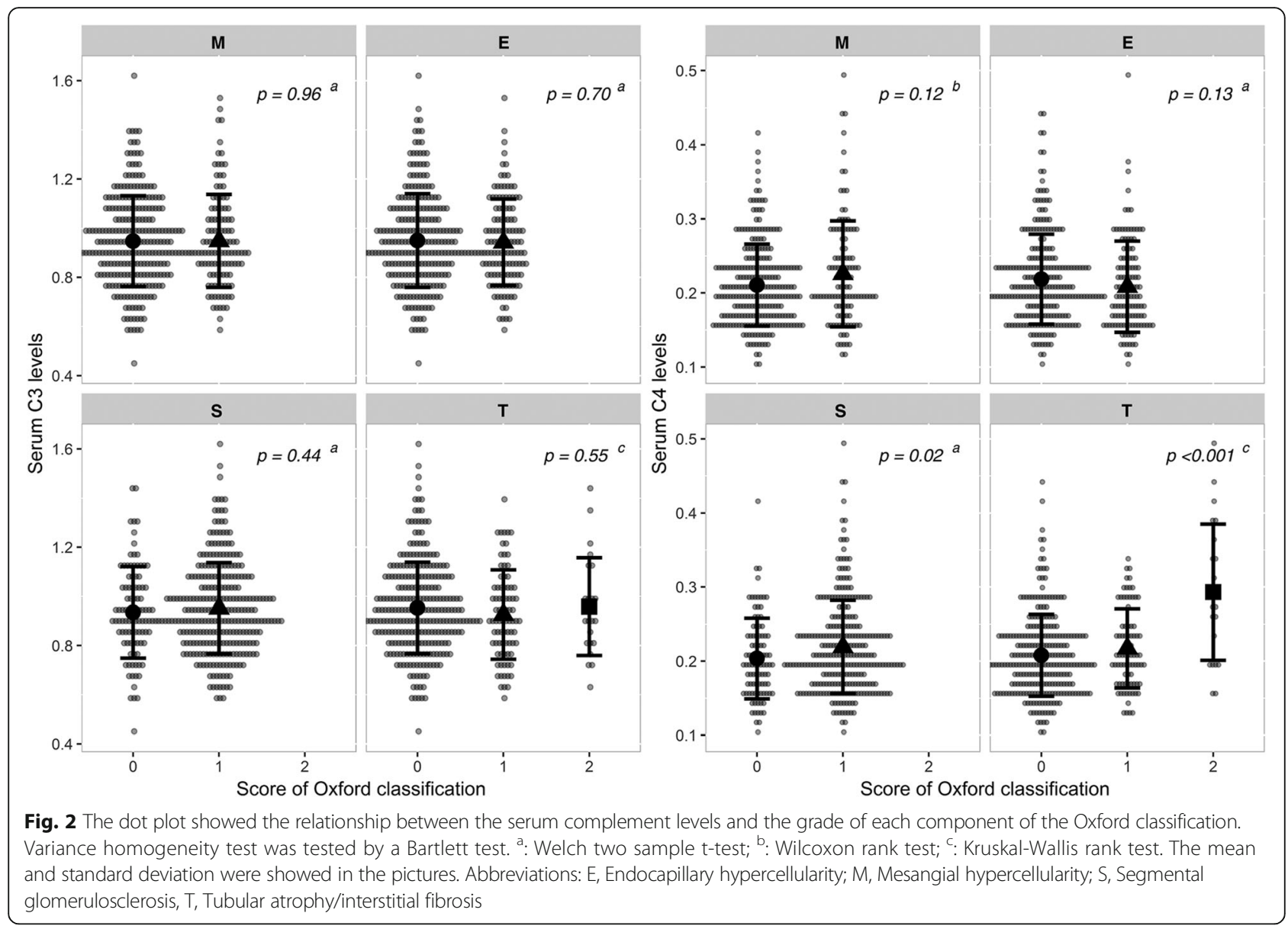

and lower $\mathrm{C} 4$ levels were related to poor prognosis, a counter-intuitive finding [14]. These studies are all limited in a variety of ways as the authors considered either $\mathrm{C} 3$ or $\mathrm{C} 4$ alone but not in combination, and this approach may therefore be inadequate for discerning the association between complement levels and renal outcomes.

Our data showed that serum C3 levels were not associated with eGFR at the time of renal biopsy, nor with those at the end of the follow-up, which was consistent

Table 3 Univariate and Multivariate Cox proportional-hazard regression analyses for the risk factors of poor outcome

\begin{tabular}{|c|c|c|c|c|}
\hline \multirow[t]{2}{*}{ Factors } & \multicolumn{2}{|c|}{ Univariate Analysis } & \multicolumn{2}{|c|}{ Multivariate Analysis } \\
\hline & $\mathrm{HR}(95 \% \mathrm{Cl})$ & $p$-value & $\mathrm{HR}(95 \% \mathrm{Cl})$ & $p$-value \\
\hline Age (Year) & $0.5(0.3-0.8)$ & 0.003 & $0.3(0.2-0.6)$ & $<0.001$ \\
\hline Gender & & 0.32 & & - \\
\hline Female & 1 & & - & \\
\hline Male & $1.4(0.7-2.6)$ & & - & \\
\hline Systolic blood pressure $(\mathrm{mmHg})$ & $1.5(1-2.2)$ & 0.04 & $1.2(0.6-2.3)$ & 0.52 \\
\hline Diastolic blood pressure $(\mathrm{mmHg})$ & $1.5(1-2.2)$ & 0.05 & $1.4(0.7-2.6)$ & 0.32 \\
\hline $24 \mathrm{~h}$ urinary protein $(\mathrm{g} / \mathrm{L})$ & $1.4(1.2-1.6)$ & $<0.001$ & $1.4(1.1-1.7)$ & 0.003 \\
\hline Serum C3 (g/L) & $0.6(0.4-0.9)$ & 0.03 & $0.4(0.2-0.6)$ & $<0.001$ \\
\hline Serum C4 (g/L) & $1.5(1-2.2)$ & 0.03 & $2.4(1.6-3.8)$ & $<0.001$ \\
\hline Immunosuppressive therapy & & 0.04 & & $<0.001$ \\
\hline GS group & 1 & & 1 & \\
\hline non-GS group & $2.0(1.1-4.0)$ & & $4.0(1.8-8.8)$ & \\
\hline
\end{tabular}




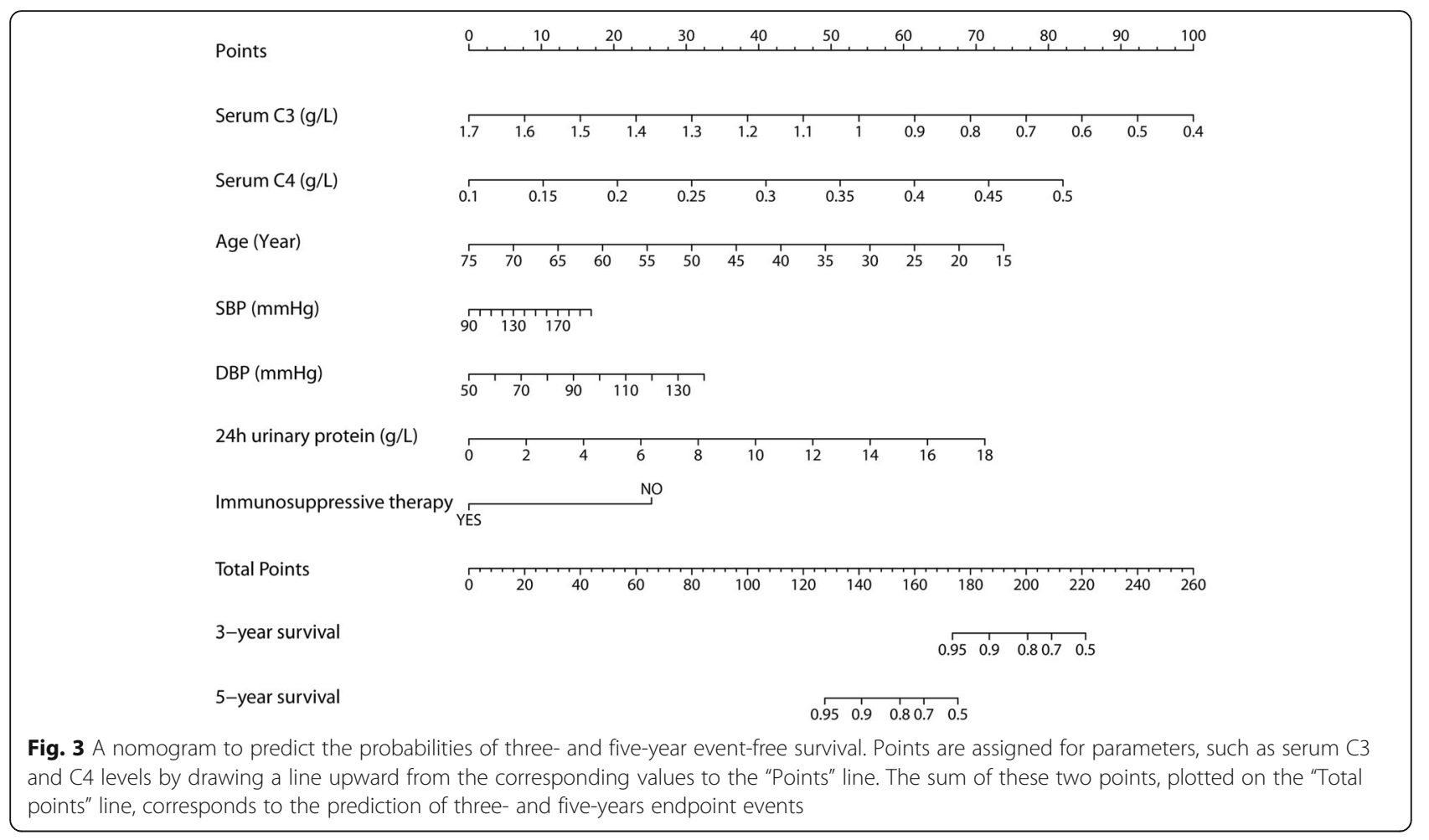

with others. On the contrary, we found that serum $\mathrm{C} 4$ levels were significantly associated with the $\mathrm{S}$ and $\mathrm{T}$ scores of the Oxford IgAN classification, and also with eGFR values. Recently, the IgAN Classification Working Group published a new classification of IgAN including crescents, called MEST-C [22]. Our data also showed that serum $\mathrm{C} 4$, but not $\mathrm{C} 3$ levels, were significantly associated with $C$ score $(P=0.04$ and $P=0.5$, respectively).

Complement components are acute phase reactive proteins, and chronic inflammation has been shown to enhance the production of complement components by hepatocytes [23]. Evidence suggests that IgAN is associated with an increase in the activation of the MBL pathway, measured as C4d and MBL deposition in glomerulus. These findings were associated with more severe of symptoms and histopathological performance [24, 25]. Espinosa et al. found that in MBL-activated IgAN, patients had a significantly higher proportion of $\mathrm{S} 1$ and $\mathrm{T} 1 / \mathrm{T} 2$ scores, but not E1 or M1 scores [24], which was consistent with our observations (Fig. 2). Furthermore, the increase in serum levels of MBL is associated with higher levels of serum complement C4 [26]. These data suggest that elevated levels of $\mathrm{C} 4$ may increase kidney damage through the MBL pathway.

Age, systolic blood pressure, diastolic blood pressure, $24 \mathrm{~h}$ urine protein are all considered to be classic risk factors of poor renal prognosis. After adjusting for these factors, the risk of adverse prognosis significantly increased by 2.5 times for every one unit decrease in serum C3 levels, and by 2.4 times for every one unit increase in serum $\mathrm{C} 4$ levels. Review of the literature suggests that the secretion, activation, and breakdown of serum complements increase during intra-renal inflammation [27]. Furthermore, serum complement levels depend upon their rate of synthesis and breakdown, which differs according to genetic background, age, the severity of disease, and the pathway of activation [21]. It is therefore reasonable that we consider the outcome-predictive effect of serum $\mathrm{C} 3$ and $\mathrm{C} 4$ together, so that the results may be more reflective of the entire picture of complement activation at the time of assay.

The limitations of our study include its retrospective nature, the short period of follow-up, and the low event number of the primary outcome, all of which potentially lead to bias. Also, there may be significant differences in the doses and the duration of immunosuppressants, the content of which were not included in the analysis. This issue can affect the predictability of serum complement levels for renal prognosis among patients with IgAN. A rigorously designed prospective study is needed to confirm our results.

\section{Conclusion}

In conclusion, increased serum $\mathrm{C} 4$ and decreased $\mathrm{C} 3$ levels at renal biopsy were associated with poor renal prognosis in patients with IgAN. Analyzing serum C3 and $\mathrm{C} 4$ levels in one model can improve the prediction of renal prognosis in these patients. 


\section{Abbreviations}

ACEl: Angiotensin-converting enzyme inhibitor; ARB: Angiotensin receptor blocker; BP: Diastolic blood pressure; C3: Serum complement 3; C4: Serum complement 4; CKD: Chronic kidney diseases; CKD-EPI: Chronic Kidney Disease Epidemiology Collaboration equation; E: Endocapillary hypercellularity; eGFR: Estimated glomerular filtration rate; ESRD: End-stage renal disease; HR: Hazard ratio; IgAN: Immunoglobulin A nephropathy; IQR: Interquartile range; M: Mesangium hypercellularity; MAP: Mean arterial pressure; MBL: Mannose-binding lectin; S: Segmental glomerulosclerosis; SD: Standard deviation; T: Tubular atrophy/interstitial fibrosis; UPCR: Urine protein to creatinine ratio

\section{Acknowledgements}

The authors would like to thank Dr. Danhua Shu for her suggestions, and colleagues in the Department of Nephrology, The Second Affiliated Hospital of Wenzhou Medical University, for their support and help during this period.

\section{Funding}

This study was supported by Wenzhou Science \&Technology Bureau (Grants Y20130208 for Ji Zhang).

\section{Availability of data and materials}

The datasets used and/or analyzed during the current study available from the corresponding author on reasonable request.

\section{Authors' contributions}

All authors have contributed significant intellectual content to this manuscript as follows: Authors' contributions All authors have contributed significant intellectual content to this manuscript as follows: Principal investigators, conception and design of the study: PM, ZJ, LZY, ZZH, LGY; Kidney specimen assessment according to the Oxford classification: $L Z Y, Z Y, J L W ;$ Assessed the studies, extracted data, performed statistical analyses: PM, Z; Drafting the manuscript: PM; Critical review: ZZH, SZ, LGY; All authors read the manuscript and approved the final version.

\section{Ethics approval and consent to participate}

This study was performed with the written informed consent of all patients and the procedure was approved by the Ethics Committee of The Second Affiliated Hospital \& Yuying Children's Hospital of Wenzhou Medical University.

\section{Consent for publication}

Not applicable.

\section{Competing interests}

The authors declare that they have no competing interests.

\section{Publisher's Note}

Springer Nature remains neutral with regard to jurisdictional claims in published maps and institutional affiliations.

\section{Author details}

'Department of Nephrology, The First Affiliated Hospital of Soochow University, 88 Shizi St., Suzhou 215006, Jiangsu, People's Republic of China. ${ }^{2}$ Department of Nephrology, The Second Affiliated Hospital \& Yuying Children's Hospital of Wenzhou Medical University, Wenzhou, Zhejiang, People's Republic of China. ${ }^{3}$ Department of Nephrology, The First Affiliated Hospital of Wenzhou Medical University, Wenzhou, Zhejiang, People's Republic of China.

Received: 26 February 2017 Accepted: 5 July 2017

Published online: 11 July 2017

\section{References}

1. Lai KN, Tang SC, Schena FP, et al. IgA nephropathy. Nat Rev Dis Primers. 2016;2:16001.

2. Donadio JV, Grande JP. IgA nephropathy. N Engl J Med. 2002;347(10):738-48.

3. Coppo R, Troyanov S, Bellur S, et al. Validation of the Oxford classification of IgA nephropathy in cohorts with different presentations and treatments. Kidney Int. 2014;86(4):828-36.

4. Barbour SJ, Espino-Hernandez G, Reich HN, et al. The MEST score provides earlier risk prediction in IgA nephropathy. Kidney Int. 2016;89(1):167-75.
5. Lhotta K, Schlogl A, Kronenberg F, Joannidis M, Konig P. Glomerular deposition of the complement C4 isotypes C4A and C4B in glomeruonephritis. Nephrol Dial Transplant. 1996;11(6):1024-8.

6. Zwirner J, Burg M, Schulze M, et al. Activated complement C3: a potentially novel predictor of progressive IgA nephropathy. Kidney Int. 1997;51(4): 1257-64.

7. Endo M, Ohi H, Ohsawa I, Fujita T, Matsushita M, Fujita T. Glomerular deposition of mannose-binding lectin (MBL) indicates a novel mechanism of complement activation in IgA nephropathy. Nephrol Dial Transplant. 1998;13(8):1984-90.

8. Ohsawa I, Ishii M, Ohi H, Tomino Y. Pathological scenario with the mannose-binding lectin in patients with IgA nephropathy. J Biomed Biotechnol. 2012;2012:476739.

9. Cattran DC, Coppo R, Cook HT, et al. The Oxford classification of IgA nephropathy: rationale, clinicopathological correlations, and classification. Kidney Int. 2009;76(5):534-45.

10. Levey AS, Stevens LA, Schmid CH, et al. A new equation to estimate glomerular filtration rate. Ann Intern Med. 2009;150(9):604-12.

11. Levey AS, Inker LA, Matsushita K, et al. GFR decline as an end point for clinical trials in CKD: a scientific workshop sponsored by the National Kidney Foundation and the US Food and Drug Administration. Am J Kidney Dis. 2014:64(6):821-35

12. Jr FEH. rms: Regression Modeling Strategies. 2016.

13. Wickham H. ggplot2: Elegant Graphics for Data Analysis. Springer-Verlag New York; 2009. http://www.springer.com/us/book/9780387981413. ISBN: 978-0-387-98140-6.

14. Zhu B, Zhu CF, Lin Y, et al. Clinical characteristics of IgA nephropathy associated with low complement 4 levels. Ren Fail. 2015;37(3):424-32.

15. Mizerska-Wasiak M, Maldyk J, Rybi-Szuminska A, et al. Relationship between serum IgA/C3 ratio and severity of histological lesions using the Oxford classification in children with IgA nephropathy. Pediatr Nephrol. 2015;30(7): 1113-20.

16. Zhang J, Wang C, Tang Y, et al. Serum immunoglobulin a/C3 ratio predicts progression of immunoglobulin a nephropathy. Nephrology (Carlton). 2013; 18(2):125-31.

17. Wada $Y$, Ogata $H$, Takeshige $Y$, et al. Clinical significance of lgG deposition in the glomerular mesangial area in patients with IgA nephropathy. Clin Exp Nephrol. 2013;17(1):73-82.

18. Kim SJ, Koo HM, Lim BJ, et al. Decreased circulating C3 levels and mesangial C3 deposition predict renal outcome in patients with IgA nephropathy. PLoS One. 2012;7(7):e40495.

19. Ibels LS, Gyory AZ. IgA nephropathy: analysis of the natural history, important factors in the progression of renal disease, and a review of the literature. Medicine (Baltimore). 1994:73(2):79-102.

20. Komatsu H, Fujimoto S, Hara S, Sato Y, Yamada K, Eto T. Relationship between serum IgA/C3 ratio and progression of IgA nephropathy. Intern Med. 2004;43(11):1023-8

21. Onda $\mathrm{K}$, Ohi $\mathrm{H}$, Tamano $\mathrm{M}$, et al. Hypercomplementemia in adult patients with IgA nephropathy. J Clin Lab Anal. 2007;21(2):77-84.

22. Trimarchi H, Barratt J, Cattran DC, et al. Oxford classification of IgA nephropathy 2016: an update from the IgA nephropathy classification working group. Kidney Int. 2017;91(5):1014-21.

23. Dean MM, Minchinton RM, Heatley S, Eisen DP. Mannose binding lectin acute phase activity in patients with severe infection. J Clin Immunol. 2005; 25(4):346-52.

24. Espinosa M, Ortega $R$, Sanchez $M$, et al. Association of $C 4 d$ deposition with clinical outcomes in IgA nephropathy. Clin J Am Soc Nephrol. 2014;9(5): 897-904.

25. Liu LL, Liu N, Chen Y, et al. Glomerular mannose-binding lectin deposition is a useful prognostic predictor in immunoglobulin a nephropathy. Clin Exp Immunol. 2013;174(1):152-60.

26. Suzuki H, Ohsawa I, Kodama F, et al. Fluctuation of serum C3 levels reflects disease activity and metabolic background in patients with IgA nephropathy. J Nephrol. 2013;26(4):708-15.

27. Onda K, Ohsawa I, Ohi H, et al. Excretion of complement proteins and its activation marker C5b-9 in IgA nephropathy in relation to renal function. BMC Nephrol. 2011;12:64. 